\title{
Oximetry use in obstructive sleep apnea
}

Félix del Campo ${ }^{1,2}$, Andrea Crespo ${ }^{1,2}$, Ana Cerezo-Hernández ${ }^{1}$, Gonzalo C Gutiérrez-Tobal ${ }^{2}$, Roberto Hornero ${ }^{2}$ and Daniel Álvarez ${ }^{*}, 1,2$

${ }^{1}$ Pneumology Service, Río Hortega University Hospital, Valladolid, Spain

${ }^{2}$ Biomedical Engineering Group, University of Valladolid, Valladolid, Spain

*Author for correspondence: dalvgon@gmail.com

\section{Funding}

This research has been partially supported by the projects DPI2017-84280-R and RTC-20153446-1 from Ministerio de Economía, Industria y Competitividad and European Regional Development Fund (FEDER), the project 66/2016 of the Sociedad Española de Neumología y Cirugía Torácica (SEPAR), and the project VA037U16 from the Consejería de Educación de la Junta de Castilla y León and FEDER. D. Álvarez was in receipt of a Juan de la Cierva grant IJCI2014-22664 from the Ministerio de Economía y Competitividad.

\section{Declaration of interest}

D. Álvarez was in receipt of a Juan de la Cierva grant IJCI-2014-22664 from the Ministerio de Economía y Competitividad. The authors have no other relevant affiliations or financial involvement with any organization or entity with a financial interest in or financial conflict with the subject matter or materials discussed in the manuscript apart from those disclosed. 


\begin{abstract}
Introduction. Overnight oximetry has been proposed as an accessible, simple, and reliable technique for obstructive sleep apnea syndrome (OSAS) diagnosis. From visual inspection to advanced signal processing, several studies have demonstrated the usefulness of oximetry as a screening tool. However, there is still controversy regarding the general application of oximetry as a single screening methodology for OSAS.
\end{abstract}

Areas covered. Currently, high-resolution portable devices combined with pattern recognitionbased applications are able to achieve high performance in the detection this disease. In this review, recent studies involving automated analysis of oximetry by means of advanced signal processing and machine learning algorithms are analyzed. Advantages and limitations are highlighted and novel research lines aimed at improving the screening ability of oximetry are proposed.

Expert commentary. Oximetry is a cost-effective tool for OSAS screening in patients showing high pretest probability for the disease. Nevertheless, exhaustive analyses are still needed to further assess unattended oximetry monitoring as a single diagnostic test for sleep apnea, particularly in the pediatric population and in especial groups with significant comorbidities. In the following years, communication technologies and big data analysis will overcome current limitations of simplified sleep testing approaches, changing the detection and management of OSAS.

Keywords: oximetry, obstructive sleep apnea syndrome, screening, signal processing, pattern recognition, machine learning 


\section{Introduction}

Obstructive sleep apnea syndrome (OSAS) is a heterogeneous chronic disease with high and increasing prevalence that commonly leads to major negative consequences for health [1]. Early diagnosis and treatment is essential to decrease the impact of OSAS in the quality of life of patients [2].

The demand for diagnostic sleep studies has significantly increased worldwide due to three major combined factors: (i) the increasing prevalence OSAS around the world $[3,4]$; the increasing awareness of patients on the negative consequences of undiagnosed disease [5]; and (iii) efficacy of therapies [6]. Nocturnal polysomnography (PSG) is the reference diagnostic method for OSAS. A positive diagnosis is confirmed when an apnea-hypopnea index (AHI) $\geq 15$ events per hour (events/h) of sleep is derived from overnight PSG or, alternatively, when an AHI $\geq 5$ events/h is obtained in the presence of common symptoms, such as excessive daytime somnolence, nonrestful sleep, daytime fatigue, and/or lack of concentration [7].

Nowadays, OSAS is considered underdiagnosed and undertreated. Unfortunately, this condition is not expected to change in the near future [8]. Despite being the reference standard technique, generalized use of PSG is still under discussion due to major limitations. It is a complex, labor-intensive, and time-consuming procedure, since specialized personnel and equipment are needed. Limited availability and accessibility to specialized outpatient facilities, sleep laboratories, and trained sleep physicians notably lead to a substantial delay in diagnosis and treatment [9].

These drawbacks have led to an increasing search for abbreviated alternative diagnostic methods. In this regard, two major improvements focus the efforts of the research and medical community: (i) from a technical point of view, the development of automated and simplified methods; and (ii) regarding patient-accessibility, the availability of portable devices able to carry out sleep studies at home. In order to achieve both goals, researchers mainly focused on the 
analysis of a reduced set of sleep-related cardiorespiratory signals, usually from portable type 3 (4 to 7 channels) or type 4 ( 1 or 2 channels, usually oximetry) devices [10]. In this regard, oximetry stands out due to its easy acquisition and interpretation.

In the present work, a systematic review of studies using pulse oximetry as a screening test for both adult and pediatric OSAS has been carried out. Science Direct, Science.gov, and PubMed were used to explore scientific/technical and biomedical research using "screening oximetry sleep apnea" as key search-term. Two independent researchers manually reviewed studies published from 2010 to 2017 . Relevant studies outside this interval were included in some areas where research is notably low, such as assessment of routine oximetry monitoring in clinical practice or the combination with additional biomedical recordings. Both Abstracts and Conference Proceedings were discarded.

\section{Technical features of oximetry devices}

Overnight oximetry has been proposed as a single diagnostic technique for OSAS due to its high accessibility, reliability, accurateness, portability, and low cost. In regard to blood oxygen saturation $\left(\mathrm{SpO}_{2}\right)$ recordings acquired with commercial pulse oximetry devices, it is important to take into account sampling frequency, time averaging, and resolution [11]. Box 1 summarizes the main utilities and technical features of pulse oximetry devices. Concerning the sampling frequency, minimum requirements are different for in-laboratory and portable devices. A sampling frequency of 25 samples per second $(\mathrm{Hz})$ is recommended by the American Academy of Sleep Medicine (AASM) for in-hospital PSG in order to perform artifact rejection (minimum 10 samples per second) [12]. However, common portable pulse oximeters just record one sample every second due to memory constrains [13]. Amplitude resolution is also an important feature. Oximetry is characterized by slow changes in $\mathrm{SpO}_{2}$ signal. Nevertheless, some authors reported that both the sampling frequency and the amplitude resolution greatly influence the measures and, 
therefore, the detection of desaturations $[14,15]$. Currently, high-resolution oximeters $(0.1 \%$ resolution) are recommended to suitably characterize oximetry dynamics. Similarly, averaging time has been found to play a major role in the shape of the $\mathrm{SpO}_{2}$ profile. Longer averaging times decrease the influence of motion artefacts. On the other hand, increasing the averaging window length leads to an underestimation of the number and severity of desaturations [16], which can be particularly relevant in some settings, such as neonatal and intensive care units [17] or at high altitude [18]. The averaging time is not standardized in available pulse oximeters [16]. Nevertheless, the AASM recommends a maximum averaging window length of 3 seconds [19].

\section{Analysis of overnight oximetry as a diagnostic tool for OSAS}

\subsection{Visual inspection and conventional indexes}

Overnight oximetry has been used as a screening test for OSAS for more than three decades. Diagnostic assessment reported in the literature is characterized by a significant variability, with sensitivity ranging from $31 \%$ to $98 \%$ and specificity ranging from 41 to $100 \%$ [20].

Visual analysis of the overnight $\mathrm{SpO}_{2}$ profile and quantification of conventional oximetric indexes based on the number and depth of desaturations have been predominantly used. Visual inspection of $\mathrm{SpO}_{2}$ searches for the common "saw tooth" pattern linked with consecutive desaturations, which is indicative of the presence of OSAS. Examination of nocturnal $\mathrm{SpO}_{2}$ tracings is still widely used and it has been recently proposed to detect OSAS in specific patients and medical circumstances, such as children [21,22] and patients with concomitant chronic obstructive pulmonary disease (COPD) [23]. The identification of this pattern is very useful for quick screening of the most severe cases, since it achieves a low false positive rate for severe OSAS. However, this technique is highly subjective and it is a time-consuming task even for trained physicians. Therefore, a thorough analysis is needed to be able to discern among nonOSAS, mild, and moderate cases. 
Both manual and computer-assisted quantification of the time, number, and severity of desaturations increased the capability of pulse oximetry to assist in the detection of OSAS. Automated algorithms have been used to estimate averages, trends, and drops characteristic of pathological patients. The most widely used measure is the oxygen desaturation index (ODI) of $2 \%$ (ODI2), 3\% (ODI3), and 4\% (ODI4), as well as the percentage of cumulative time (CT) from the whole recording with a saturation below a predetermined threshold, usually $90 \%$ (CT90).

\subsection{Advanced signal processing and automated pattern recognition}

In the context of automated signal processing and pattern recognition, the following stages are essential to compose and optimize a high-performance as well as generalizable model [24]:

- Feature extraction, in order to obtain as much information as possible from the signal.

- Feature selection, aimed at removing redundant information and maximizing complementarity of relevant data.

- Pattern recognition, in order to design and optimize a classifier aimed at assisting in the detection of OSAS.

Figure 1 shows common stages in automated signal processing of oximetry. Additionally, usual outputs of pattern recognition techniques in the context of OSAS detection are listed in Box 2.

In recent years, several studies have been carried out focusing on automated signal processing of oximetry in the framework of OSAS diagnosis. In this regard, features from both time and frequency domains provided essential information on the dynamics of oximetry [25]. Morphological features [26] and statistics from the data histogram [25] have been found to be very useful. Similarly, due to the repetitive behavior of apneic/hypopneic events, oximetric 
recordings of positive-OSAS patients show a significant power increase in certain frequency bands $[15,25,27,28]$.

A major contribution in the context of OSAS diagnosis from oximetry was the use of nonlinear methods to characterize changes in the dynamics of overnight $\mathrm{SpO}_{2}$ recordings due to apneic events. In this regard, entropy, central tendency measure, and Lempel-Ziv complexity have been used to quantify irregularity, variability, and complexity of the oximetry signal, respectively $[15,25,29]$. Entropy is commonly used to characterize the dynamics of complex biological systems, so that the higher the entropy the higher the irregularity. In the framework of oximetry and OSAS, there is a close relation between entropy, ODI3, and the AHI $[25,29,30]$. It is important to point out that different metrics have been proposed to estimate entropy. In a recent study, Marcos et al. [31] carried out a comparative assessment of approximate entropy (ApEn), sample entropy (SampEn), and kernel entropy (KernEn) in the context of adult OSAS diagnosis. They reported that KernEn reached the highest diagnostic performance, although significantly higher computational time is required. Similarly, multiscale entropy (MSE) has recently been found to be relevant and complementary to assist in pediatric OSAS diagnosis using unattended oximetry at home [32].

The central tendency measure (CTM) is a nonlinear measure of variability, so that the lower the CTM the higher the variability of the signal. Different studies reported significantly decreased CTM values, i.e., higher variability, in $\mathrm{SpO}_{2}$ recordings from OSAS patients due to the recurrent desaturations, both in adults [29] and in children [15,24]. Similarly, the Lempel \& Ziv complexity (LZC) is a nonlinear measure of complexity linked with the number of new patterns and their repetition along the signal. Increased LZC is related to the presence of more new patterns, i.e., higher complexity. In the context of OSAS, high LZC is linked with higher complexity in the $\mathrm{SpO}_{2}$ signal of OSAS patients due to the common desaturations [25]. 
In the context of advanced signal processing, Schlottahuer et al. [33] assessed the usefulness of empirical mode decomposition (EMD) in order to characterize overnight oximetry in OSAS patients. EMD decomposes a time series into different components called intrinsic mode functions, which are modulated in amplitude and frequency to gather all the relevant information present in the signal. Using this technique, the authors estimated the ODI from intrinsic mode functions, reaching a high and balanced sensitivity-specificity pair (83.8\%vs. 85.5\%).

In the last decade, application of computers and novel technologies in the field of medicine and healthcare increased exponentially. Particularly, computer-aided diagnosis and predictive models have significantly increased the diagnostic ability of oximetry in the context of OSAS. Several pattern recognition techniques have been proposed in the literature. The research in the context of OSAS mainly focuses on binary classification in order to detect the presence or absence of the disease. Similarly, some researchers apply multiclass techniques to classify each patient into one of the common OSAS severity groups, namely non-OSAS, mild, moderate, and severe. On the other hand, in spite of a better characterization of the severity of the disease, few studies have been conducted that were aimed at estimating the AHI by means of regression approaches.

\section{Automated classification of oximetric patterns}

\subsection{Binary and multiclass approaches}

Regarding conventional statistical classifiers, linear discriminant analysis (LDA) and logistic regression (LR) have been widely applied in the context of OSAS diagnosis from oximetry, whereas quadratic discriminant analysis (QDA) has been marginally used [24]. On the other hand, high-performance techniques such as artificial neural networks (ANNs) are reliable alternatives. ANNs allow researchers to analyze nonlinear relationships commonly present in biological systems. They are mathematical models inspired in the information processing capabilities of human neurons (parallel processing, distributed memory/storage, and 
environmental flexibility) and they have been widely applied in the context of OSAS. There is not a predetermined network architecture known to be a priori the best for any problem under study. Nevertheless, the multilayer perceptron (MLP) is probably the most widely used approach due to its simplicity as well as high performance [24].

Table 1 summarizes the main characteristics of studies using pattern recognition techniques for classification of suspected OSAS patients. In the study by Almazaydeh et al. [34], a MLP ANN was proposed to perform binary classification of OSAS patients from overnight oximetry recordings. The network was fed with the conventional ODI3 and the delta index, as well as a nonlinear measure of variability by means of the CTM. The Authors reported $87.5 \%$ sensitivity, $100 \%$ specificity, and $93.3 \%$ accuracy in a validation set from PhysioNet, a publicly available and widely used dataset.

Morillo et al. [35] optimized a probabilistic ANN (PNN) fed with time-domain indices (desaturations and resaturations $\geq 3 \%$ and $4 \%$ ), frequency-domain features (power spectral density, PSD), stochastic (CTs in standardized saturation intervals), and nonlinear measures (Poincaré plots). Binary classification was performed, considering AHI $\geq 10$ as positive OSAS. The authors reported notably high diagnostic accuracy (93.91\%) with balanced sensitivity $(92.42 \%)$ and specificity $(95.92 \%)$.

An alternative pattern recognition approach also used to detect OSAS from oximetry are support vector machines (SVMs). SVMs are high-performance classifiers able to reach high generalization both in classification and regression problems [24]. In this regard, Álvarez et al. [29] compared the diagnostic performance obtained with SVM, LDA, and LR in this context. Feature selection techniques were applied to a wide initial set of statistics in the time and frequency domains, conventional spectral features, and nonlinear measures in order to obtain an optimum feature subset from oximetry. SVM reached the maximum diagnostic performance (84.2\% accuracy) in the first independent test set and maintained the accurateness $(84.5 \%$ 
accuracy) in a second test set. LR reached the maximum performance though accuracy notably varied between validation sets $(83.2 \%$ vs. 88.7$)$.

Recently, Hang et al. [36] used a SVM-based classifier to assess the usefulness of oximetry as screening test for adult OSAS. Conventional ODI2 and ODI4 were used to parameterize oximetry. A 4-class SVM classifier reached $71.27 \%$ overall accuracy for the prediction of OSAS severity (no-OSAS, mild, moderate, and severe) in a dataset composed of 616 patients. Regarding binary classification, a SVM reached $90.42 \%$ accuracy and 0.954 area under the ROC curve (AUC) in the detection of severe OSAS (AHI $\geq 30$ events/h), whereas $87.33 \%$ accuracy and 0.921 AUC were reached for moderate-to-severe OSAS (AHI $\geq 15$ events $/ \mathrm{h}$ ). Adding clinical variables to ODIs, such as body mass index (BMI), neck circumference (NC) or Epworth sleepiness scale (ESS), did not lead to a significant performance increase. Individually, single ODI4 reached similar accuracy than SVM classifiers but lower AUC.

Huang et al. [37], assessed decision trees (DTs), MLP ANNs, learning vector quantization (LVQ), and adaptive network-based fuzzy inference systems (ANFIS), as automated methods for OSAS screening using ODI4. The proposed DT reached $94.67 \%$ accuracy $(98.67 \%$ sensitivity and 90.67 specificity), outperforming MLP (90.67\% accuracy), ANFIS (88.33\% accuracy), and LVQ (80.00\% accuracy).

\subsection{Estimation of the AHI using oximetry}

There are few studies aimed at estimating the actual PSG-based AHI using oximetric data. Nevertheless, this is an important goal since clinicians directly use the AHI as marker of the severity degree as well as to manage treatment in their daily practice. Furthermore, describing OSAS severity by means of AHI is needed in Medicare and Medicaid services both for diagnosis and for reimbursement [38]. ODIs can be directly used as a substitute of the AHI in order to estimate the number of respiratory events during the night. However, it is widely known that ODIs 
underestimate the severity of OSAS notably. Conversely, regression models can be used to properly estimate the AHI. In this regard, it is widely known that the duration of desaturations does not correlate well with the actual duration of respiratory events. However, studies aimed at estimating the AHI using oximetry alone do not focus on the detection and quantification of every single respiratory event. Machine learning algorithms, such as artificial neural networks, support vector machines, or regression trees, process input variables derived from the whole overnight oximetry profile (desaturation indexes, overall statistics, spectral, and nonlinear measures) to optimize a model able to provide an accurate estimate of the actual AHI. Table 2 summarizes the main characteristics of studies focused on AHI estimation from oximetry.

Marcos et al. [39] carried out a study aimed at estimating the AHI using a MLP ANN fed with spectral and nonlinear features from attended in-lab oximetry. A total of 240 patients showing suspicion of OSAS were involved in the study. The proposed network reached an intraclass correlation coefficient (ICC) of 0.91 . Furthermore, using a cut-off of 15 events/h for moderate-to-severe OSAS, the authors reported $94.87 \%$ sensitivity, $90.91 \%$ specificity, and $93.1 \%$ accuracy.

In the study by Ebben \& Krieger [40] a regression model for AHI estimation based on ODI4 was developed. Four different approaches were assessed: Linear, multivariate adaptive splines, segmented, and quadratic regression. The highest performance was obtained by means of quadratic regression, reaching an ICC of 0.88 (0.87-0.90). Regarding binary classification, accuracies ranged from $91 \%$ to $95 \%$ using the common cut-offs for positive OSAS. Similarly, in a recent study by Jung et al. [26], the AHI was estimated by means of nonlinear Hill regression using oximetry data. Firstly, a rough estimation of respiratory events was made using morphological information from the overnight oximetric profile (depth and duration of desaturations). Then, a Hill regression model was optimized using the number of apneic events per hour of recording as dependent variable. ICC of 0.99 and mean absolute error of 2.30 events/h 
were obtained. An average diagnostic accuracy of $96.7 \%$ was reached using the common cut-offs for the disease.

\subsection{Oximetry and ensemble learning}

In order to improve the diagnostic performance, some authors recently focused on the combination of several classifiers, the so-called ensemble learning. Table 3 summarizes the main characteristics of studies using ensemble learning techniques to assist in OSAS diagnosis using oximetry.

Sánchez-Morillo et al. [41] optimized an ensemble of classifiers composed of decision trees (DTs), SVMs, and PNNs fed with statistical, spectral, and nonlinear features from oximetry. The classifier was aimed at categorizing patients into the four common severity groups (no-SASH, mild, moderate, and severe). A population composed of 115 patients with high pre-test probability of suffering from OSAS were analyzed. Diagnostic accuracy of $86.0 \%$ and 0.8 agreement were reported using leave-one-out cross-validation.

Hang et al. [42] also assessed an ensemble of binary SVMs in the context of OSAS detection from oximetry. In addition, feature selection by means of genetic algorithms (GAs) was used in order to obtain the most predictive features and to optimize the parameters of the ensemble. An ensemble composed of 10 SVM binary classifiers achieved the highest accuracy $(90.37 \pm 0.71 \%)$ for severe OSAS, slightly outperforming the accuracy reached by single SVMs.

Similalry, Xie et al. [43] optimized an automated classifier based on data from oximetry and ECG in order to detect apneic events in real time. A total of 111 features from ECG both in the time and in the frequency domains, as well as 39 oximetric features including conventional oximetric indexes, statistics and nonlinear measures, were used. The authors reported a maximum accuracy of $86.6 \%$ using an ensemble of decision trees. However, sensitivity and specificity were 
notably unbalanced. Lower imbalance was achieved using a classifier composed of 3 experts with subsequent majority voting. Nevertheless, overall accuracy was slightly lower and computational time increased significantly.

\section{Oximetry-based applications for simplified detection and management of OSAS}

\subsection{Portable oximetry in OSAS patients with comorbidities and special risk groups}

In order to expedite diagnosis, researchers have assessed several screening approaches for OSAS based on portable monitoring. However, there is a lack of studies evaluating the effectiveness of unattended oximetry at patient's home in the presence of comorbidities. Some works have been carried out, particularly in stroke patients [44] and patients with heart failure [45] or arterial hypertension [46]; in the presence of morbid obesity [47] or chronic obstructive pulmonary disease (COPD) [23,48]; in patients remitted for surgery [49]; or in frail hospitalized elderly patients [50]. Table 4 summarizes the main characteristics of studies using oximetry to assist in OSAS diagnosis in patients with comorbidities.

OSAS is a common sleep-related breathing disorder in stroke patients, which is linked with decreased recovery as well as increased risk of mortality [44]. In this regard, Aaronson et al. [44] assessed the diagnostic accuracy of oximetry to detect OSAS in patients in stroke rehabilitation. An ODI4 $\geq 15$ events/h reached $77 \%$ sensitivity and $100 \%$ specificity in the prediction of an AHI $\geq 15$ events $/ \mathrm{h}$. The authors reported that nocturnal oximetry alone is able to accurately diagnose moderate-to-severe OSAS in stroke patients.

In the study carried out by Ward et al. [45], conventional ODI3 from unattended portable monitoring was used for screening for OSAS in patients with heart failure. The authors individually assessed the diagnostic performance of the ODI3 from oximetry and the percent very low frequency increment (\%VLFI) from HRV. It is noteworthy that HRV time series were measurable only in $45 \%$ of patients, while oximetry was valid in $99 \%$ of the population under 
study. An ODI3 > 7.5 desaturations/h achieved high sensitivity (97\%) and negative predictive value (94\%) to rule out the disease, although specificity (32\%) and positive predictive value (53\%) were poor. Overall, the diagnostic performance of ODI3 was high (0.92 AUC; $93 \%$ sensitivity and $73 \%$ specificity for a cut-off of $>12.5$ ). On the other hand, the diagnostic accuracy of \%VLFI was extremely poor (0.50 AUC), achieving $58 \%$ sensitivity and $48 \%$ specificity for a cut-off of $\geq 2.23$.

Regarding arterial hypertension, the European Respiratory Society recommends screening for OSAS in hypertensive patients showing symptoms of sleep disorders [51]. In this way, Maricoto et al. [46] recently assess nocturnal oximetry in patients with uncontrolled hypertension. Rigorous criteria based on ODIs from oximetry were used to confirm (with $100 \%$ sensitivity) or discard (with $100 \%$ specificity) the disease in a sample of hypertensive patients. Then, ambulatory blood pressure monitoring was used to evaluate blood pressure control. The authors concluded that nocturnal portable pulse oximetry might be a good screening test for OSAS in patients with uncontrolled hypertension.

Malbois et al. [47] used oximetry alone as abbreviated screening test for OSAS in morbid obese patients before bariatric surgery. Focusing on severe OSAS patients (AHI $\geq 30$ events/h), the authors reported $100 \%$ sensitivity and $93 \%$ specificity using the conventional ODI3 obtained via unsupervised polygraphy at home (type III portable monitoring). Similarly, Chung et al. [49] assessed ODI4 from portable pulse oximetry as predictor of the AHI in surgical patients. A total of 475 patients were involved in the study, showing an average BMI of $31 \pm 7 \mathrm{~kg} / \mathrm{m}^{2}$. An ODI4 $\geq 5,15$, and 30 reached AUC of $0.908,0.931$, and 0.958 in the prediction of mild, moderate-tosevere, and severe OSAS, respectively. The authors concluded that high-resolution oximetry is a sensitive as well as specific tool to detect OSAS in patients referred for surgery. Nevertheless, obesity [47] and overweight [49] might influence the results in both studies. 
Overnight oximetry has been also assessed as screening test for OSAS in the elderly. In the study by Mazière et al. [50], an automated variability index was derived from the nocturnal oximetric profile of 58 hospitalized patients older than 75 years old. An index greater than 0.51 reached perfect sensitivity $(100 \%)$ for moderate-to-severe OSAS, whereas specificity was significantly lower (18.8\%). Conventional ODI3 and ODI4 were more specific but less sensitive than the proposed variability index.

Scott et al. [23] carried out a retrospective study to test the usefulness of oximetry in patients with COPD. An earlobe portable pulse oximeter was assessed in the hospital to determine the presence or absence of OSAS (actual AHI $\geq 15$ events/h) in 59 COPD patients. The study was aimed at developing general criteria for visual interpretation of the oximetric profile by non-expert personnel. Visual inspection of oximetry reached a sensitivity of $59 \%$ and a specificity of $60 \%$, whereas the performance slightly increased up to $60 \%$ sensitivity and $63 \%$ specificity using the automated ODI4. The authors concluded that oximetry provided modest diagnostic performance in identifying moderate-to-severe OSAS in patients with moderate-to-severe COPD.

Similarly, Andrés et al. [48] recently assessed the effectiveness of oximetry as diagnostic tool for OSAS in the presence of COPD. The authors characterized overnight oximetry by means of statistical, spectral, and nonlinear measures, which fed a regression MLP ANN aimed at estimating the AHI. The proposed methodology was assessed both at home and in the laboratory and two independent validation datasets were analyzed: (i) COPD and (ii) non-COPD. The oximetry-based ANN reached similar ICC values between the estimated and actual AHI for the non-COPD and the COPD groups both in the hospital (0.937 vs. 0.936) and at home (0.731 vs. 0.788). Notably high diagnostic performance was reached in both settings, particularly for severe OSAS: 0.97 vs. 0.98 AUC in the hospital and 0.87 vs. 0.86 at home for non-COPD and COPD patients, respectively. The Authors concluded that oximetry could be used as a simplified screening test for moderate-to-severe OSAS regardless the presence of COPD. 


\subsection{Oximetry in clinical practice}

Despite its theoretical usefulness as abbreviated screening test particularly for severe patients, few studies assess oximetry in clinical practice. In this regard, Epton et al. [52] recently found that general practitioners skilled in the diagnosis and management of sleep disordered breathing were able to improve the accuracy of referrals for subsequent specialized assessment and potential treatment. Studies assessing oximetry-based approaches to assist in OSAS diagnosis in clinical practice are summarized in Table 5.

Chai-Coetzer et al. [11] assessed an abbreviated two-stage screening test in a population attending primary care for any reason. Firstly, sleep questionnaires were used as screening stage for moderate-to-severe OSAS. Then, the ApneaLink was used to monitor oximetry and airflow at home in order to derive ODI3 and estimate the AHI. At-home PSG was used as reference standard. The two-stage model reached an overall diagnostic accuracy of $83 \%$, as well as notably high NPV (96\%) but poor PPV (56\%). The screening ability of ODI3 from oximetry alone was higher than the AHI estimated using both oximetry and airflow. The authors concluded that the proposed protocol could potentially offer a cost-effective tool to manage OSAS and expedite diagnosis particularly in rural and remote regions. In a recent study, Chai-Coetzer et al. [53] analyzed the influence of abbreviated sleep studies on physician decision making and patient outcomes. Particularly, the authors compared scores from sleep-related questionnaires, CPAP compliance, and physician diagnostic confidence in patients referred to standard PSG versus patients referred to cardiorespiratory polygraphy and pulse oximetry. No statistically significant differences were found in patient outcomes when cardiorespiratory polygraphy was carried out. On the other hand, major evidences were obtained regarding potential diminished outcomes from patients using pulse oximetry. Less improvement in Epworth sleepiness scale, sleep apnea symptoms questionnaire, and CPAP use were reported, as well as lower physician diagnostic confidence. 
Antic et al. [54] carried out a randomized study in tertiary centers aimed at assessing a simplified management protocol of continuous positive airway pressure (CPAP) treatment at patient's home. Patients showing high probability of moderate-to-severe OSAS according to athome overnight oximetry and reported symptoms (ODI2 >27 events/h, snoring, and ESS >8) were randomly assigned to 2 models of care: 1) a simplified nurse-led model using at-home autoadjusting CPAP; 2) a physician-lead model involving conventional PSG both to diagnose and to set the therapy optimal pressure of the device. The simplified nurse-led model showed noninferior results compared to the conventional model while being less expensive. Similarly, Sharma et al. [55] assessed a simplified model in hospitalized obese patients at a tertiary academic center in order to expedite early treatment and assess survival in relation to adherence. In this study, an ODI4 $\geq 5$ desaturations/h predicted mild-to-severe OSAS with an AUC of 0.83 , showing notably higher sensitivity (89\%) than specificity $(48 \%)$. Their results suggested that oximetry is a useful screening method to detect undiagnosed sleep disordered breathing in hospitalized obese patients.

\subsection{Comparative studies}

There are few studies comparing oximetry-based approaches with other simplified methods aimed at assisting in OSAS diagnosis. Recent approaches are summarized in Table 6. Rofail et al. [56] compared airflow from nasal cannula with oximetry, both recorded at patient's home during 3 consecutive nights. In-hospital PSG was the gold standard. The authors found that no significant differences exist between both simplified approaches in terms of diagnostic performance and that no improvement is obtained using both recordings jointly. On the other hand, it is noteworthy that a 3-night testing at home reached higher diagnostic accuracy than one night alone. In a study carried out by Ting et al. [57], a comparison among oximetry, airflow, and pulse rate variability signals simultaneously recorded was developed involving Taiwanese male drivers. Oximetry reached the best screening ability, particularly when actigraphy was used to estimate the total 
sleep time. Similarly, Shih et al. [58] used overnight high-resolution oximetry as marker of OSAS in order to assess long-term cardiovascular risk in drivers.

Dawson et al. [59] developed a comparative study between ApneaLink and conventional oximetry alone using in-hospital PSG as gold standard. A small population was recruited and recordings showed several artifacts and signal loss. Similar AUC values were reached using ODI4 from oximetry (0.827) and ApneaLink (0.840). Therefore, the authors concluded that airflow from ApneaLink did not provide significant further information to oximetry in the context of adult OSAS.

\subsection{Combination with complementary biomedical signals}

Data derived from nocturnal $\mathrm{SpO}_{2}$ recordings and additional sleep-related signals have been jointly analyzed in order to improve diagnostic performance. Table 7 shows recent studies.

Barak-Shinar et al. [60] used $\mathrm{SpO}_{2}$ and photoplethismography (PPG) signals derived from in-hospital PSG in order to estimate the AHI. Sensitivity and specificity were both higher than 90\% in the detection of mild and moderate-to-severe OSAS. Similarly, Yadollahi et al. [61] combined oximetry and tracheal sounds in order to detect individual apnea and hypopnea events and subsequently obtain an estimation of the AHI. Both signals were recorded during in-hospital PSG, which was considered the gold standard. The authors reported $84.6 \%$ sensitivity and $96.0 \%$ specificity for moderate-to-severe OSAS.

Recently, Li et al. [62] carried out a study aimed at assessing PPG from oximetry in OSAS diagnosis. The authors recorded the PPG signal of 49 patients in a hospital setting using portable monitoring simultaneously to PSG. Amplitude changes in the PPG signal were used to obtain the PPG-derived respiration waveform. Apneic events were quantified when changes in the PPGbased respiration were linked with oxygen desaturations. A respiratory events index was 
quantified accordingly. The proposed index reached higher correlation with actual AHI from PSG, achieving $89.7 \%$ sensitivity and $90.0 \%$ specificity in the prediction of moderate OSAS. Lower performance and unbalanced sensitivity and specificity were reached for mild and severe cases.

Oximetric variables have been combined with cardiac information derived from the ECG $[43,63,64]$. Alternatively, pulse rate from peripheral PPG has been used mainly in children due to its lower intrusiveness [15]. Regarding adult OSAS, Henegan et al. [63] developed a prospective study (59 adult patients) combining ECG and oximetry (Holter-oximeter). Both signals were recorded in the hospital simultaneously to standard PSG. An AHI was automatically computed and compared with manual AHI from PSG, achieving 95.8\% sensitivity and 100\% specificity (LR+ >20) for mild OSAS. Automated and manual AHIs were significantly correlated. As previously discussed, Xie et al. [43] reported 86.6\% accuracy using features from oximetry and ECG by means of ensemble learning methods.

Oximetry has been marginally used to detect the presence of central respiratory events. To achieve this goal, Abedi et al. [65] proposed to parameterize oximetry and thoracic effort by means of time-domain, frequency-domain, and nonlinear features. Genetic algorithms (GAs) were applied for feature selection while a SVM was used for classification. The authors reported $96.0 \%$ accuracy in a patient-oriented classification (normal vs. OSAS), while 87.8\% accuracy was reached in the detection of obstructive and central apneas. The performance discerning between obstructive and central episodes increased using oximetry or respiratory effort individually (accuracy $>90 \%$ ) but unbalanced sensitivity and specificity was obtained.

\subsection{Oximetry as screening tool for pediatric OSAS}

As in the case of adult patients, in-hospital PSG is also the gold standard method for a definitive diagnosis of OSAS in children. However, limitations regarding accessibility and intrusiveness of PSG are even more significant in pediatric patients [66]. In this regard, recent 
reports of major sleep/respiratory international societies $[66,67]$ highlight the need for further research on screening tests for the disease, as well as the development of simplified tools able to increase accessibility to diagnostic resources. Standard PSG will be limited to complex cases were simplified techniques do not provide sufficiently high certainty or when conventional complex techniques are needed to set the treatment modality, e.g., non-invasive ventilation $v s$. surgery. In this context, oximetry has been also proposed as an appropriate technique due to its simplicity and readiness.

Overall, the amount of research on the usefulness of oximetry to assist in pediatric OSAS diagnosis is limited compared with adult OSAS. Conventional indexes such as ODIs have been predominantly assessed while the application of advanced signal processing and pattern recognition techniques increased during the last years. Studies assessing oximetry-based approaches to assist in pediatric OSAS diagnosis are summarized in Tables 8 and 9.

Velasco-Suarez et al. [68] reported that the presence of two or more clusters of desaturations $\geq 4 \%$ from baseline as well as desaturation events below $90 \%$ predicted childhood OSAS with $86.6 \%$ sensitivity and $98.9 \%$ specificity by visual inspection of nocturnal oximetric profiles. The authors concluded that visual analysis of oximetry is a simple and useful technique in the diagnosis and management of children showing clinical suspicion of OSAS secondary to adenotonsillar hypertrophy. Similarly, Van Eyck et al. [22] and Villa et al. [69] assessed the diagnostic performance of clusters of desaturations in the context of childhood OSAS. In [22], diagnostic accuracies ranging 68-78\% were obtained using a cut-off point of 2 events/h for positive OSAS. In order to improve performance, Villa et al. [69] combined clusters of desaturations and clinical history, achieving $85.8 \%$ and $69.4 \%$ accuracies for cut-offs of 1 and 5 events/h, respectively.

The combination of children's symptoms with ODI also focused the interest of researchers in order to design a high-performance screening methodology for OSAS. Chang et al [70] 
analyzed a population composed of 141 children in 2013. They found that increased ODI, witnessed apneas, mouth breathing, and restless sleep were correlated with AHI. The authors proposed an interesting scoring in order to manage the disease. Firstly, when apneas are observed, PSG is directly proposed to obtain definitive diagnosis and treatment. When no apneas are observed, mouth breathing (+1 point), restless sleep $(+1$ point $)$ and oximetry $(1<\mathrm{ODI}<3:+1$ point; ODI $\geq 3:+2$ points) are considered: (i) children with a score $\leq 1$ are considered normal although should be monitored; (ii) children with a score equal to 2 are derived to PSG; (iii) children with a score $\geq 3$ are considered OSAS-positive and referred to a specialist. Using this protocol, $84 \%$ positive predictive value was achieved.

Tsai et al. [71] also assessed conventional ODI as predictor of childhood OSAS. They retrospectively analyzed 146 habitually snoring children with suspicion of suffering from the disease. ODI4 significantly correlated with AHI $(0.886 ; p<0.001)$. The best cut-off point for detecting at least mild OSAS (AHI $\geq 1$ ) was ODI4 $\geq 2.05$ desaturations/h, which provided a significant screening ability for rule in the disease (98.1\% PPV).

Recently, Jing-Ru et al. [72] assessed a pulse oximetry watch (POW) for diagnosing pediatric OSAS. A population composed of 32 children referred due to snoring were selected. PSG and portable oximetry were carried our simultaneously in the hospital. No significant differences between ODI4 from PSG and ODI4 from POW were found. For common cut-offs for mild $(A H I \geq 1)$, moderate $(A H I \geq 5)$, and severe $(A H I \geq 10)$ pediatric OSAS, ODI4 from portable oximetry showed poor performance, achieving AUC $<0.80$ and sensitivities below $65 \%$. The best diagnostic ability was reached using a notably high cut-off of 20 events/h, obtaining $83.33 \%$ sensitivity, 92.31\% specificity, and 0.929 AUC.

In order to increase accessibility and availability of diagnostic tools in pediatric OSAS, Garde et al. [15] developed the Phone Oximeter, which is a portable device integrating a pulse oximeter with a smartphone. The Phone Oximeter is able to record both oximetry and pulse rate 
derived from the photoplethismographic wave. The authors recruited a population composed of 146 children in order to assess its effectiveness for moderate-to-severe OSAS diagnosis (AHI $\geq 5$ events/h). Using the Phone Oximeter in the hospital, the variability of $\mathrm{SpO}_{2}$ and the signal power at low frequencies were significantly higher $(p<0.01)$ in children showing OSAS. In addition, the analysis of PRV showed a significant increase of sympathetic activity due to intermittent hypoxia in OSAS-positive children. Using oximetry alone, a linear discriminant model achieved balanced sensitivity and specificity ( $88.4 \%$ vs. $83.6 \%$, respectively) as well as notably high negative predictive value $(92.6 \%)$ and 0.82 AUC. Furthermore, the authors found that combining information from $\mathrm{SpO}_{2}$ and $\mathrm{PRV}$, the model reached higher diagnostic ability (0.88 AUC). Cohen \& De Chazal [73] also combined oximetry and information from heart rate. They analyzed unattended portable recordings of 288 children in order to detect and quantify individual apneic events. The authors reported $74.7 \%$ diagnostic accuracy in an event-based classification using time and frequency features from the ECG signal. The accuracy decreased up to $66.7 \%$ when oximetric features from the time domain were included in the model.

Last year, our own group carried out a study aimed at assessing automated analysis of athome unattended oximetry in the context of childhood OSAS [28]. In this research, 50 children with high pre-test probability of OSAS underwent nocturnal respiratory polygraphy (RP) at home, whereas conventional in-hospital PSG was used as gold standard. Statistical, spectral, and nonlinear features were computed from oximetric recordings derived from unattended RP. A logistic regression model was optimized by means of GAs. Diagnostic accuracies of $85.5 \%$ (0.97AUC), 83.4\% (0.96 AUC), and 82.8\% (0.97 AUC) were reached using AHI $\geq 1,3$, and 5 events/h as cut-offs for positive OSAS. The optimum LR model significantly outperformed manual AHI from RP for a cut-off of 1 event/h, whereas similar diagnostic performance was reached using the remaining cut-offs. Additionally, conventional ODI3 was significantly outperformed regardless the cut-off for pediatric OSAS. Gutiérrez-Tobal et al. [74] analyzed the same dataset in a previous study, where ODI3 was combined with spectral features from airflow, 
both obtained during unattended RP at home. A LR model achieved 86.3\% accuracy (0.947 AUC) using a cut-off of 3 events/h, significantly improving ODI3 and airflow alone.

Recently, Crespo et al. [32] investigated the dynamics of unsupervised oximetry in order to build high-performance predictors of pediatric OSAS. In this regard, MSE was used to characterize nonlinear patters present in the oximetric profile caused by apneic events. A LR model composed of both entropy-based features and conventional oximetric indices automatically selected reached $83.5 \%$ accuracy (0.86 AUC).

Regarding the usefulness of novel signal processing techniques and their capability to provide complementary information to traditional approaches, a recent study carried out by Vaquerizo-Villar et al. [75] assessed bispectrum as an alternative to conventional PSD. Bispectrum is able to detect deviations from linearity, stationarity, and gaussianity, commonly present in biomedical signals. A multiclass MLP ANN fed with bispectral, PSD-derived, ODI3, and anthropometric variables reached high accuracies in the detection of moderate-to-severe (AHI $\geq 5$ events/h: $81.3 \%$ accuracy) and severe (AHI $\geq 10$ events/h: $85.3 \%$ accuracy) childhood OSAS.

In 2017, a multicenter international study leaded by our group and the University of Chicago prospectively analyzed the usefulness of oximetry as abbreviated screening test for pediatric OSAS [76]. A total of 13 pediatric sleep laboratories were involved worldwide and 4,191 habitually snoring children were recruited. To our knowledge, this is the largest sample analyzed in this context. Oximetry was derived from in-hospital PSG and subsequently analyzed by means of a regression MLP ANN aimed at estimating the AHI. It is important to highlight that most studies focuses on binary classification of children as having pediatric OSAS or not. On the contrary, this study provides an estimation of the AHI just using overnight oximetry. In addition, only two oximetric variables feed the ANN, ODI3 and the third-order moment in the frequency domain, thus becoming a really simple as well as accurate approach. High agreement with actual AHI from PSG was reported (0.785 ICC). The assessment of common AHI-based cutoffs revealed 
an incremental diagnostic ability: 75.2\% (0.788 AUC), 81.7\% (0.854 AUC), and 90.2\% (0.913

AUC) accuracy was reached for $\mathrm{AHI} \geq 1,5$, and 10 events/h, respectively.

\subsection{Oximetry portable monitoring by means of smartphones}

In order to increase accessibility to diagnosis, some authors have proposed to use smartphones. Nowadays, smartphones integer several sensors able to record, store, and even analyze different biomedical signals, such as snoring or movements. Moreover, medical accessories have been developed to be connected to the smartphone via common integrated ports in order to acquired additional signals, such as ECG and oximetry. In this regard, Behar et al. [77] developed an application to screen for OSAS using a smartphone. The authors stated that snoring sounds (audio) and movements (actigraphy) could be recorded using built-in sensors, whereas oximetry may be potentially obtained using a portable commercial oximeter connected to the smartphone via Bluetooth. However, in this study, audio, actigraphy and photoplethysmography were recorded by means of polygraphy carried out at home. Multiscale entropy was used to parameterize audio and actigraphy, whereas the PPG signal was used to compute the ODI. In addition, clinical variables (BMI, age, neck size, and gender) were used. The App used a SVMbased classifier to screen for the disease, achieving $92.3 \%$ accuracy in the detection of moderateto-severe OSAS versus being healthy or habitually snorer (mild OSAS patients were excluded).

The use of smartphones is also interesting in the context of pediatric OSAS in order to minimize the intrusiveness of conventional techniques for children. In this regard, the Phone Oximeter developed by Garde et al. [15] is able to obtain oximetric and pulse rate features from overnight recordings using a smartphone. The authors developed an application integrating a signal processing model that reached higher diagnostic ability in the detection of childhood OSAS. Nevertheless, recordings were obtained in the laboratory. Therefore, further validation of the whole system is needed at home. 


\section{Expert commentary}

OSAS is a very prevalent illness showing a heterogeneous symptom presentation, which ranges from low clinical suspicion to evident limitations in the quality of life. The main reasons for this variability relate to its potential association with major concomitant diseases, which include diabetes and a variety of cardiovascular pathologies. Current treatment modalities for OSAS have shown to be able to diminish most of the negative consequences of the disease. However, there is an important bottleneck concerning the availability and accessibility to diagnostic resources. In this context, the usefulness of pulse oximetry as a simplified test for OSAS arises. Significant scientific evidences have been reported concerning the high screening ability of oximetry, particularly when it is used with specific patient profiles [78]. Notwithstanding this consideration, well-known limitations exist, which merit further research.

Most of the studies evaluating the utility of pulse oximetry have been conducted involving subjects with moderate-to-high suspicion of OSAS. Consequently, its usefulness has to be assessed in more detail in mild cases. In addition, the high variability in the diagnostic performance of oximetry reported in these studies seems to corroborate the need for more specific research. In this sense, research lines such as those focused on the night-to-night variability, the influence of unattended signal acquisition on cost-effectiveness of OSAS diagnosis or the technical features of the recording devices should be addressed.

Regarding ambulatory sleep studies, single-channel approaches for sleep apnea detection mainly focus on the simplification of the diagnostic methodology in order to increase availability, decrease cost, minimize intrusiveness, and allow for screening testing at home. On the other hand, focusing on the use of oximetry alone, important limitations arise when comprehensive respiratory analyses are needed. Additional respiratory effort-based signals (thoracic and/or abdominal) would be needed to accurately discern among obstructive, central, and mixed apnea episodes. Similarly, there are medical conditions that could influence the performance of automated 
decision-making systems based solely on oximetry, particularly the presence of concomitant respiratory diseases such as COPD or hypoventilation syndromes. In this regard, there is an increasing demand for further assessment of abbreviated screening tests for OSA detection in the presence of comorbidities $[79,80]$. Recent studies demonstrated the usefulness of automated analysis of oximetry in the detection of OSA in patients with COPD [48]. Nevertheless, further research is still needed to definitively validate single-channel oximetry as a reliable alternative to standard PSG in the screening of OSA in patients with special medical conditions.

Regarding the pulse oximeter setting, statistically significant differences in the results derived from different devices have been reported [14], most probably due to their technical specifications rather than to the detection algorithms used to compute oximetry indexes, such as ODIs [81]. In recent years, a number of new pulse oximeters have been launched. These novel devices include improved features, such as high resolution and sample frequency, as well as an enhanced output in artifact and low perfusion conditions $[49,82]$. However, very few studies have been devoted to the comparison of the performance among these new devices $[14,15,49,58]$.

Other important issue to be noted is that there is no consensus regarding the definition and scoring of a desaturation event. It is also not clear which of the desaturation indexes (ODI3 or ODI4) is more helpful. While ODI3 may show higher correlation with AHI, ODI4 might show a stronger relation with arterial hypertension development. Hence, a gap is still present in the study of commonly used clinical parameters and their specific usefulness.

In recent years, researchers have focused on the evaluation of unattended oximetry in order to increase the accessibility to diagnosis. The usefulness of oximetry maximizes when the diagnostic test is conducted at home. It is clear that reproducing the same conditions in which patients sleep every night is the best way to test for sleep-related breathing disorders. However, many studies used in-laboratory techniques carried out in a different night as the gold standard, predominantly PSG. Therefore, the validation of portable oximetry at home against the reference 
diagnostic method conducted in the same unattended setting simultaneously is another interesting research line that has not been properly addressed.

One of the latest advances in the assessment of pulse oximetry is the incorporation of novel automatic signal processing and machine learning techniques aimed at increasing accuracy and assisting in the automation of the diagnostic process. A significant number of studies have been published in the last 15 years not only in engineering and technical journals, but also in the framework of respiratory and sleep medicine. The implementation of signal processing and machine learning methods is a complex task. Nevertheless, the computational time is negligible once the algorithms have been developed. Most of these studies implement a common standardized methodological approach. Firstly, an initial feature extraction stage is conducted, in which oximetry-derived signals are exhaustively characterized. Then, a feature selection stage is (implicitly or explicitly) implemented to select the most relevant and complementary variables, which maximize the pattern-recognition ability of the information (oximetric features) obtained in the previous step [29]. Finally, these optimum features are used to train a given machine learning algorithm with ability to carry out a classification or a regression task. In the first case, the algorithm will categorize patients into predefined classes, assisting physicians in decisionmaking. Patients can be classified into OSAS-positive or OSAS-negative, if the algorithm was trained to predict the presence or absence of the disease (binary classification). It is also possible to determine the severity group, i.e. non-OSAS, mild, moderate, or severe classes, if the model was designed to provide a severity degree (multiclass classification). In the regression case, the aim is to estimate the AHI using oximetry alone. In addition, different cut-offs can be subsequently set to perform binary or multiclass classification.

In the context of AHI regression, it is widely known that the duration of desaturations does not correlate well with the actual duration of respiratory events. However, studies aimed at estimating the AHI using oximetry alone do not focus on the detection and quantification of every 
single respiratory event. Machine learning algorithms, such as artificial neural networks, support vector machines, or regression trees, process input variables derived from the whole overnight oximetry profile (desaturation indexes, overall statistics, spectral and nonlinear measures) to optimize a model able to provide an accurate estimate of the actual AHI.

A proper use of automated signal processing and pattern recognition techniques can overcome the limitations of conventional ODI3 and ODI4 in the framework of simplified OSAS detection. Oximetric indices are known to achieve high specificity but lower sensitivity. On the other hand, machine learning approaches are able to reach notably higher sensitivity values while maintaining a balanced specificity. However, important precautions should be taken when training machine learning methods in order to obtain reliable models, particularly regarding the size of the training dataset. Similarly, independent validation procedures need to be implemented at every stage of the methodology, one for each degree-of-freedom (user-dependent parameter) during the design of the predictive models. In addition, most of the machine learning-based classification approaches tend to favor the class with the largest number of instances (subjects). Hence, balancing the proportion of subjects of each class during training may dramatically improve models performance.

Automated signal processing and pattern recognition of overnight oximetry signals can provide simple as well as accurate diagnostic tools alternative to standard PSG, leading to more timely treatment and potentially improved outcomes. However, it is important to highlight that there is still some discrepancy concerning the effectiveness of portable monitoring (and, particularly, pulse oximetry alone) as a single tool for OSAS detection. A recent study found no major changes in patient outcomes and physician confidence in the diagnoses when using cardiorespiratory polygraphy. Additionally, a significantly lower improvement in symptoms and CPAP compliance, as well as lower diagnostic confidence were reported when using pulse oximetry alone, primarily in milder cases [53]. Similarly, data supporting simplified monitoring 
at home for the diagnosis of OSAS in children is currently insufficient. Recent reports $[83,84]$ point out that overnight pulse oximetry is far from being a perfect screening tool for mild OSAS and specific subgroups of children. It should be noted that advanced signal processing algorithms, such as ensemble and deep learning techniques, could provide more reliable results and help to overcome oximetry drawbacks.

The results from studies analyzed in this review suggest that oximetry could be a costeffective tool for OSAS screening in patients showing a high pretest probability for the disease. Nevertheless, exhaustive analyses are still needed to further assess unattended oximetry monitoring as a single diagnostic test for sleep apnea, particularly in the pediatric population and in especial groups with significant comorbidities, in order to overcome current limitations and improve the quality of care.

\section{Five-year view}

In the following years, communication technologies and, particularly, health-related applications for smartphones will enable real implementation of unsupervised screening and monitoring of OSAS at home. In the near future, novel machine learning algorithms and big data analysis will change the detection and management of chronic diseases. In the context of OSAS, high-performance portable oximeters with high sampling frequency, amplitude resolution, memory storage, and battery life will contribute to provide continuous information on the patient's health status in order to enable personalized medicine.

Leading scientific societies in the context of sleep medicine currently demand further research focused on pediatric patients and subjects with comorbidities. Deep learning algorithms from big data analysis will contribute to identify such particular subpopulations where the diagnostic ability of oximetry could be maximized. 


\section{Key issues}

- Obstructive sleep apnea syndrome is a highly prevalent yet relatively underdiagnosed condition able to significantly decrease health and quality of life.

- Availability and accessibility to sleep laboratories is limited and the gold standard diagnostic method is complex, intrusive, and labor-intensive.

- Overnight oximetry has been extensively assessed as a simplified screening test for the disease due to its simplicity, availability, portability, and significant effectiveness.

- Conventional oxygen desaturation indexes reached moderate-to-high screening ability for ruling in severe obstructive sleep apnea, although there is still not a standardized definition of desaturation.

- Signal processing techniques are able to further characterize oximetry dynamics. Morphological and statistical features in the time domain, spectral features, and nonlinear measures have demonstrated significant combined relevancy.

- Automated pattern recognition techniques have been found to increase the diagnostic performance of oximetry. Conventional statistical modes, artificial neural networks, and more recently support vector machines and ensemble learning have provided incremental diagnostic ability.

- There are robust evidences regarding the validity of oximetry as screening test in a clinical setting. Nevertheless, further research is still needed to support oximetry monitoring at home as a single diagnostic test for sleep apnea, particularly in children and especial groups with significant comorbidities. 


\section{References}

Papers of particular significance are highlighted as "of interest" (*) or "of considerable interest" $(* *)$.

[1] Peppard PE, Young T, Barnet JH, Palta M, Hagen EW, Hla KM. Increased prevalence of sleep-disordered breathing in adults. Am. J. Epidemiol. 177(9), 1006-1014 (2013).

[2] Sharma S, Chowdhury A, Tang L, Willes L, Glynn B, Quan SF. Hospitalized patients at high risk for obstructive sleep apnea have more rapid response system events and intervention is associated with reduced events. PLoS One. 11(5), e0153790 (2016).

[3] Senaratna CV, Perret JL, Lodge CJ, et al. Prevalence of obstructive sleep apnea in the general population: A systematic review. Sleep Med. Rev. 34, 70-81 (2017).

[4] Peppard PE, Young T, Barnet JH, Palta M, Hagen EW, Hla KM. Increased prevalence of sleep-disordered breathing in adults. Am. J. Epidemiol. 177(9), 1006-1014 (2013).

[5] Ayas NT, Taylor CM, Laher I. Cardiovascular consequences of obstructive sleep apnea. Curr. Opin. Cardiol. 31(6), 599-605 (2016).

[6] Walter RJ, Hagedorn SI, Lettieri CJ. Impact of diagnosing and treating obstructive sleep apnea on healthcare utilization. Sleep Medicine. 38, 73-77 (2017).

[7] American Academy of Sleep Medicine. International classification of sleep disorders. 3rd edition. American Academy of Sleep Medicine; Darien, IL (2014).

[8] Lorenzi-Filhoa G, Gentaa PR, Drage LF. Are we missing obstructive sleep apnea diagnosis? Rev. Port. Pneumol. 23(2), 55-56 (2017).

[9] Readline S. Screening for obstructive sleep apnea. Implications for the sleep health of the population. JAMA. 317(4), 368-370 (2017).

[10]Collop NA, Tracy SL, Kapur V, et al. Obstructive sleep apnea devices for out-of-center (OOC) testing: technology evaluation. J. Clin. Sleep Med. 7(5), 531-548 (2011). 
[11]Chai-Coetzer CL, Antic NA, Rowland LS, et al. A simplified model of screening questionnaire and home monitoring for obstructive sleep apnoea in primary care. Thorax. 66(3), 213-219 (2011).

[12] Iber C, Ancoli-Israel S, Chesson AL. Quan SF for the American Academy of Sleep Medicine. The AASM Manual for the scoring of sleep and associated events. Rules, terminology and technical specifications. 1st edition. American Academy of Sleep Medicine; Westchester, IL (2007).

[13]Pretto JJ, Roebuck T, Beckert L, Hamilton G. Clinical use of pulse oximetry: Official guidelines from the Thoracic Society of Australia and New Zealand. Respirology. 9, 38-46 (2014).

[14]Böhning N, Schultheiß B, Eilers S, Penzel T, Böhning W, Schmittendorf E. Comparability of pulse oximeters used in sleep medicine for the screening of OSA. Physiol. Meas. 31, 875888 (2010).

[15]Garde A, Dehkordi P, Karlen W, Wensley D, Ansermino JM, Dumont GA. Development of a screening tool for sleep disordered breathing in children using the Phone OximeterTM. PLoS One. 9, e112959 (2014).

** This paper assesses a portable device integrating a pulse oximeter with a smartphone in the context of childhood OSAS. Blood oxygen saturation and pulse rate are automatically analyzed.

[16] Vagedes J, Bialkowski A, Wiechers C, Poets CF, Dietz K. A conversion formula for comparing pulse oximeter desaturation rates obtained with different averaging times. PLoS ONE 9(1), e87280 (2014).

[17] McClure C, Young JS, Fairchild K. Alarms, oxygen saturations, and $\mathrm{SpO} 2$ averaging time in the NICU. Journal of Neonatal-Perinatal Medicine, 9(4), 357-362 (2016). 
[18] Cross TJ, Keller-Ross M, Issa A, Wentz R, Taylor B, Johnson B. The impact of averaging window length on the "desaturation" indexes obtained via overnight pulse oximetry at high altitude. SLEEP. 38(8),1331-1334 (2015).

[19] Berry RB, Budhiraja R, Gottlieb DJ, et al. Rules for scoring respiratory events in sleep: update of the 2007 AASM Manual for the Scoring of Sleep and Associated Events. J. Clin. Sleep Med. 8(5), 597-619 (2012).

[20]Uddin MB, Chow CM, Su SW. Classification methods to detect sleep apnea in adults based on respiratory and oximetry signals: a systematic review. Physiol. Meas. 39(3), 03TR01 (2018).

[21] Velasco-Suarez CT, Figueroa-Turienzo JM, Len F, Mansilla E. Pulse oximetry recording in children with adenotonsillar hypertrophy: usefulness in the diagnostic of obstructive sleep apnea syndrome. Arch. Argent. Pediatr. 111, 196e201 (2013).

[22] Van Eyck A, Lambrechts C, Vanheeswijck L. The role of nocturnal pulse oximetry in the screening for obstructive sleep apnea in obese children and adolescents. Sleep Med. 16, 1409$1412(2015)$.

[23] Scott AS, Baltzan MA, Wolkove N. Examination of pulse oximetry tracings to detect obstructive sleep apnea in patients with advanced chronic obstructive pulmonary disease. Can. Respir. J. 21, 171-175 (2014).

[24] Álvarez D, Cerezo-Hernández A, López-Muñiz G, et al. Usefulness of Artificial Neural Networks in the Diagnosis and Treatment of Sleep Apnea-Hypopnea Syndrome, Sleep Apnea - Recent Updates, Rijeka, Croacia: InTech, Editores: Mayank G. Vats. ISBN: 978-953-513055-0 (2017).

[25] Álvarez D, Hornero R, Marcos JV, Del Campo F. Feature selection from nocturnal oximetry using genetic algorithms to assist in obstructive sleep apnoea diagnosis. Med Eng Phys. 34(8), 1049-1057 (2012). 
[26]Jung DW, Hwang SH, Cho JG, et al. Real-Time Automatic Apneic Event Detection Using Nocturnal Pulse Oximetry. IEEE Trans. Biomed. Eng. In press (2017).

[27]Hang LW, Chen YF, Lin ChL. Frequency domain index of oxyhemoglobin saturation from pulse oximetry for obstructive Sleep Apnea Syndrome. J. Med. Biol. Eng. 32(5), 343-348 (2012).

[28] Álvarez D, Alonso-Álvarez ML, Gutiérrez-Tobal GC, et al. Automated screening of children with obstructive sleep apnea using nocturnal oximetry: An alternative to respiratory polygraphy in unattended settings. J. Clin. Sleep Med. 13(5), 693-702 (2017).

[29] Álvarez D, Hornero R, Marcos JV, et al. Assessment of feature selection and classification approaches to enhance information from overnight oximetry in the context of apnea diagnosis, Int. J. Neural Syst. 23(05), 1350020 (2013).

[30]Crespo A, Álvarez D, Kheirandish-Gozal L, et al. Assessment of oximetry-based statistical classifiers as simplified screening tools in the management of childhood obstructive sleep apnea Sleep Breath. In Press, (2018).

[31] Marcos JV, Hornero R, Nabney R, Alvarez D, Gutiérrez-Tobal G, del Campo F. Regularity analysis of nocturnal oximetry recordings to assist in the diagnosis of sleep apnoea syndrome. Med. Eng. Phys. 38, 216-224 (2016).

[32] Crespo A, Álvarez D, Gutiérrez-Tobal GC, et al. Multiscale Entropy Analysis of Unattended Oximetric Recordings to Assist in the Screening of Paediatric Sleep Apnoea at Home. Entropy. 19, 284 (2017).

[33] Schlotthauer G, Di Persia LE, Larrateguy LD. Screening of obstructive sleep apnea with empirical mode decomposition of pulse oximetry. Med. Eng. Phys. 36, 1074-1080 (2014).

[34] Almazaydeh L, Faezipour M, Elleithy KA. Neural network system for detection of obstructive sleep apnea through $\mathrm{SpO} 2$ signal features. International Journal of Advanced Computer Science and Applications. 3, 7-11 (2012). 
[35] Morillo DS, Gross N. Probabilistic neural network approach for the detection of SAHS from overnight pulse oximetry. Med. Biol. Eng. Comput. 51(3), 305-315 (2013).

[36]Hang LW, Wang HL, Chen JH, et al. Validation of overnight oximetry to diagnose patients with moderate to severe obstructive sleep apnea. BMC Pulm. Med. 15, 24-37 (2015).

[37]Huang S-H, Teng N-C, Wang K-J, Chen K-H, Lee H-C, Wang P-C. Use of oximetry as a screening tool for obstructive sleep apnea: a case study in Taiwan. J Med. Sys. 39, 29 (2015).

[38]Department of Health and Human Services, Centers for Medicare \& Medicaid Services. Continuous and bi-level positive airway pressure devices: complying with documentation and coverage requirements (2013).

[39] Marcos JV, Hornero R, Álvarez D, Aboy M. Del Campo F. Automated prediction of the apnea-hypopnea index from nocturnal oximetry recordings. IEEE Trans. Biomed. Eng. 59, 141-149 (2012).

** This paper assesses the diagnostic performance of a regression neural network aimed at estimating the apnea-hypopnea index using oximetry alone. Time, spectral, and nonlinear features from oximetric recordings fed the neural network.

[40]Ebben MR and Krieger AC. Diagnostic accuracy of a mathematical mode to predict apneahypopnea index using night time pulse oximetry. J. Biomed. Optics. 21(3), 035006 (2016).

[41]Sánchez-Morillo D, López-Gordo MA, León A. Novel multiclass classification for homebased diagnosis of sleep apnea hypopnea syndrome. Expert Systems with Applications. 41, 1654-1662 (2014).

[42]Hang LW, H-H Lin, JY Chiang, H-L Wang, Y-F Chen. Diagnosis of Severe Obstructive Sleep Apnea with Model Designed Using Genetic Algorithm and Ensemble Support Vector Machine. Appl. Math. 7(1S), 227S-336S (2013).

[43] Xie B, Minn H. Real-time sleep apnea detection by classifier combination. IEEE Trans. Inf. Technol. Biomed. 16, 469-477 (2012). 
** This paper assesses several pattern recognition techniques for real-time sleep apnea detection. High performance is obtained using ensemble learning with statistical, time, and frequency features from oximetry and ECG.

[44]Aaronson JA, Van Bezeij T, Van den Aardweg JG, Van Bennekom CAM, Hofman WF. Diagnostic accuracy of nocturnal oximetry for detection of sleep apnea syndrome in stroke rehabilitation. Stroke. 43(9), 2491-2493 (2012).

[45] Ward NR, Cowie MR, Rosen SD, Roldao V, De Villa M. Utility of overnight pulse oximetry and heart rate variability analysis to screen for sleep-disordered breathing in chronic heart failure. Thorax. 67(11), 100-105 (2012).

[46] Maricoto T, Silva EAR, Damião P, Bastos JM. The OXIMAPA Study: Hypertension Control by ABPM and Association with Sleep Apnea Syndrome by Pulse Oximetry. Acta Med. Port. 30(2), 93-99 (2017).

[47] Malbois M, Giusti V, Suter M, Pellaton C, Vodoz J-F, Heinzer R. Oximetry alone versus portable polygraphy for sleep apnea screening before bariatric surgery. Obesity Surgery. 20(3), 326-331 (2010).

[48] Andrés-Blanco MA, Álvarez D, Crespo A, et al. Assessment of automated analysis of portable oximetry as a screening test for moderate-to-severe sleep apnea in patients with chronic obstructive pulmonary disease. PLoS ONE. 12(11), e0188094 (2017).

** A recent paper assessing the effectiveness of automated analysis of portable oximetry to detect sleep apnea in COPD patients. The apnea-hypopnea index estimated using a regression neural network fed with oximetric features is assessed in the hospital and at home.

[49]Chung F, Liao P, Elsaid H, Islam S, Shapiro CM, Sun Y. Oxygen desaturation index from nocturnal oximetry: a sensitive and specific tool to detect sleep- disordered breathing in surgical patients. Anesth Analg. 114(5), 993-1000 (2012).

[50] Mazière S, Pepin JL, Siyanko N, et al. Usefulness of oximetry for sleep apnea in frail hospitalized elderly. JAMDA. 15, 447e9-14 (2014). 
[51]Parati G, Lombardi C, Hedner J, et al. Recommendations for the management of patients with obstructive sleep apnoea and hypertension. Eur. Respir. J. 41, 523-538 (2013).

[52] Epton MJ, Kelly PT, Shand BI, et al. Development and outcomes of a primary carebased sleep assessment service in Canterbury, New Zealand. npj Primary Care Respiratory Medicine. 27, 26 (2017).

* A recent paper evaluating a community-based sleep assessment service carried out by general practitioners skilled in sleep disordered breathing. A protocol based on multidisciplinary meeting and overnight oximetry is proposed.

[53] Chai-Coetzer CL, Antic NA, Hamilton GS, et al. Physician decision making and clinical outcomes with laboratory polysomnography or limited-channel sleep studies for obstructive sleep apnea. Ann. Intern. Med. 166, 332-340 (2017).

[54] Antic NA, Buchan C, Esterman A, et al. A randomized controlled trial of nurse-led care for symptomatic moderate-severe obstructive sleep apnea. Am. J. Respir. Crit. Care Med. 179(6), 501-508 (2009).

[55] Sharma S, Mukhtar U, Kelly C, Mather P, Quan SF, Recognition and treatment of sleep disordered breathing in obese hospitalized patients may improve survival. The HoSMed database, The American Journal of Medicine. In press (2017).

[56]Rofail LM, Wong KK, Unger G, Marks GB, Grunstein RR. Comparison between a singlechannel nasal airflow device and oximetry for the diagnosis of obstructive sleep apnea. Sleep. 33(8), 1106-1114 (2010).

[57]Ting H, Huang R-J, Lai C-H, et al. Evaluation of Candidate Measures for Home-Based Screening of Sleep Disordered Breathing in Taiwanese Bus Drivers. Sensors. 14, 81268149 (2014).

[58] Shih T-S, Liou S-H. Utility of overnight pulse oximeter as a screening tool for sleep apnea to assess the 8-year risk of cardiovascular disease: Data from a large-scale bus driver cohort study. International Journal of Cardiology. 225, 206-212 (2016). 
[59]Dawson A, Loving RT, Gordon RM et al. Type III home sleep testing versus pulse oximetry: is the respiratory disturbance index better than the oxygen desaturation index to predict the apnoea-hypopnoea index measured during laboratory polysomnography? BMJ Open. 5, e007956 (2015).

[60]Barak-Shinar D, Amos Y, Bogan RK. Sleep disordered breathing analysis in a general population using standard pulse oximeter signals. Sleep Breath. 17(3), 1109-1115 (2013).

[61] Yadollahi A, Giannouli E, Moussavi Z. Sleep apnea monitoring and diagnosis based on pulse oximetry and tracheal sound signals. Med. Biol. Eng. Comput. 48, 1087-1097 (2010).

[62] Li Y, Gao H, Ma Y. Evaluation of pulse oximeter derived photoplethysmographic signals for obstructive sleep apnea diagnosis. Medicine. 96, 18 (2017).

[63] Heneghan C, Chua CP, Garvey JF, et al. A portable automated assessment tool for sleep apnea using a combined holter-oximeter. SLEEP. 31(10), 1432-1439 (2008).

[64]Poupard L, Philippe C, Goldman MD, Sartne R, Mathieu M. Novel mathematical processing method of nocturnal oximetry for screening patients with suspected sleep apnoea syndrome. Sleep Breath. 16(2), 419-425 (2012).

[65] Abedi Z, Naghavi N, Rezaeitalab F. Detection and classification of sleep apnea using genetic algorithms and SVM-based classification of thoracic respiratory effort and oximetric signal features. Computational Intelligence. 33, 1005-1018 (2017).

* This is a recent research aimed at assessing the joint analysis of overnight oximetry and respiratory effort recordings in order to develop improved screening test for OSAS. Both event- and patient-oriented classification is proposed using a SVM for classification.

[66] Marcus CL, Brooks LJ, Ward SD, et al. Diagnosis and management of childhood obstructive sleep apnea syndrome. Pediatrics. 130, e714-755 (2012).

[67] Kaditis AG, Alonso-Alvarez ML, Boudewyns A, et al. Obstructive sleep disordered breathing in 2- to 18-year-old children: diagnosis and management. Eur. Respir. J. 47, 69-94 (2016). 
[68] Velasco-Suarez CT, Figueroa-Turienzo JM, Len F, Mansilla E. Pulse oximetry recording in children with adenotonsillar hypertrophy: usefulness in the diagnostic of obstructive sleep apnea syndrome. Arch. Argent. Pediatr. 111, 196e201 (2013).

[69] Villa MP, Pietropaoli N, Supino MC, et al. Diagnosis of pediatric obstructive sleep apnea syndrome in settings with limited resources. JAMA. Otolaryngol. Neck Sur. 141, 990-996 (2015).

[70]Chang L, Wu J, Cao L. Combination of symptoms and oxygen desaturation index in predicting childhood obstructive sleep apnea. Int. J. Pediatr. Otorhinolaryngol. 77, 365-371 (2013).

[71]Tsai CM, Kang $\mathrm{CH}$, Su MC, et al. Usefulness of desaturation index for the assessment of obstructive sleep apnea syndrome in children. Int. J. Pediatr. Otorhinolaryngol. 77, 12861290 (2013).

[72]Jing-Ru M, Jing-Jing H, Qi C, Hai-Tao W, Kuan-Lin X, Yu-Tian Z. Value of pulse oximetry watch for diagnosing pediatric obstructive sleep apnea/hypopnea syndrome. Acta OtoLaryngologica. 1-6 (2017).

[73] Cohen G, de Chazal P Automated detection of sleep apnea in infants: A multi-modal approach. Comput. Biol. Med. 63, 118-123 (2015).

[74] Gutiérrez-Tobal GC, Alonso-Álvarez ML, Álvarez D, Del Campo F, Terán-Santos J, Hornero R. Diagnosis of pediatric obstructive sleep apnea: Preliminary findings using automatic analysis of airflow and oximetry recordings obtained at patients' home. Biomed. Signal Process. Control. 18, 401-407 (2015).

[75] Vaquerizo-Villar F, Álvarez D, Kheirandish-Gozal L, et al. Utility of bispectrum in the screening of pediatric sleep apnea-hypopnea syndrome using oximetry recordings. Comput. Meth. Prog. Biomed. 156, 141-149 (2018). 
[76] Hornero R, Kheirandish-Gozal L, Gutiérrez-Tobal GC, et al. Nocturnal Oximetrybased Evaluation of Habitually Snoring Children. Am. J. Respir. Crit. Care Med. 196, 1591-1598 (2017).

** A recent multicenter study assessing overnight oximetry as a simplified screening tool for pediatric OSAS. To our knowkedge, this is the study with the largest sample investigating automated pattern recognition of oximetric recordings in the context of sleep apnea in children.

[77]Behar J, Roebuck A, Shahid M, et al. SleepAp: An Automated Obstructive Sleep Apnoea Screening Application for Smartphones. IEEE J Biomed. Health Inf. 19(1), 325-331 (2015).

[78] Thurnheer R. Diagnostic approach to sleep-disordered breathing. Expert Rev. Respir. Med. 5(4), 573-589 (2011).

[79] Treptow E, Oliveira MG, Moreira GA, Togeiro S, Nery LE, Tufik S, Bittencourt L. Update on the use of portable monitoring system for the diagnosis of sleep apnea in specific population. World J Respirol 2015; 5(1): 17-27.

[80] Cooksey JA, Balachandran JS. Portable monitoring for the diagnosis of OSA. Chest. 2016; 149:1074-81.

[81]Ng Y, Joosten SA, Edwards BA, et al. Oxygen Desaturation Index Differs Significantly Between Types of Sleep Software. J. Clin. Sleep Med. 13(4), 599-605 (2017).

[82] Sharma S, Mather PJ, Chowdhury A, et al. Sleep overnight monitoring for apnea in patients hospitalized with heart failure (SOMA-HF Study). J. Clin. Sleep Med. 13(10), 1185-1190 (2017).

[83] Annelies Van Eyck \& Stijn L. Verhulst. Improving the diagnosis of obstructive sleep apnea in children with nocturnal oximetry-based evaluations, Expert Rev. Respir. Med. In press (2018). 
[84]Kirk V, Baughn J, D’Andrea L, et al. American Academy of Sleep Medicine position paper for the use of a home sleep apnea test for the diagnosis of OSA in children. J. Clin. Sleep Med. 13(10), 1199-1203 (2017). 


\section{Figure legends}

Figure 1. Common stages of automated signal processing and pattern recognition approaches applied to oximetry in the context of OSAS detection.

ANNs: artificial neural networks; CTs: cumulative times; DTs: decision trees; FSLR: forward stepwise logistic regression; GAs: genetic algorithms; LDA: linear discriminant analysis; LR: logistic regression; ODIs: oxygen desaturation indices; PCA: principal component analysis; PSD: power spectral density; QDA: quadratic discriminant analysis; STFT: short-time Fourier transform; SVM: support vector machines; SWLDA: stepwise linear discriminant analysis. 